\title{
Oclusão Percutânea de Defeitos Cardíacos Congênitos e Estruturais com Amplatzer Duct Occluder II ${ }^{\mathrm{TM}}$
}

\author{
Marcelo S. Ribeiro ${ }^{1,2}$, Fabricio L. Pereira ${ }^{1,2}$, Rodrigo N. Costa ${ }^{1,2}$, Airton Arruda ${ }^{3}$, Sérgio Braga ${ }^{1,2}$, \\ Valmir F. Fontes ${ }^{1,2}$, Carlos A. C. Pedra ${ }^{1,2}$
}

\section{RESUMO}

Introdução: Uma nova geração de próteses Amplatzer com menor perfil e maior flexibilidade foi desenvolvida recentemente para o tratamento percutâneo da persistência do canal arterial (PCA). Neste artigo é relatado o uso desse novo dispositivo, Amplatzer Duct Occludder II ${ }^{\mathrm{TM}}$ (ADO II), para o tratamento tanto de PCA como de outros defeitos congênitos e estruturais, avaliando sua eficácia e segurança. Métodos: Estudo longitudinal observacional de uma coorte de pacientes portadores de cardiopatias congênitas e estruturais tratados com ADO II entre outubro de 2009 e agosto de 2011. Pacientes com menos de $5 \mathrm{~kg}$ e canais $>5,5 \mathrm{~mm}$ e/ou do tipo B foram excluídos do estudo. A prótese foi implantada por vias anterógrada ou retrógrada. A cintura foi cerca de $2 \mathrm{~mm}$ maior que o defeito e o comprimento da prótese foi de $4 \mathrm{~mm}$ para lesões mais curtas (até 6-8 $\mathrm{mm}$ ). Resultados: Foram incluídos no estudo 28 pacientes, com mediana de idade e de peso de 2 anos e $11,7 \mathrm{~kg}$, respectivamente, dos quais 23 eram portadores de PCA com diâmetro mínimo de $2,3+0,8 \mathrm{~mm}$. Os demais apresentavam comunicação interventricular muscular (1), fístula arteriovenosa pulmonar (1), aneurisma roto do seio de Valsalva (1), e coração univentricular em pós-operatório recente de cavopulmonar bidirecional com fluxo anterógrado pulmonar residual (2). Em todos os pacientes a prótese foi implantada com sucesso, com exceção de um lactente de $8 \mathrm{~kg}$ com canal arterial de 2,8 $\mathrm{mm}$ de diâmetro, de ampola rasa e de trajeto longo, tortuoso e oblíquo. Houve oclusão total dos defeitos em todos os pacientes. Um paciente portador de coração univentricular e isomerismo direito faleceu em decorrência de complicações não associadas ao procedimento. Conclusões: A prótese ADO II mostrou-se versátil, segura e eficaz no tratamento percutâneo de lactentes, crianças e adultos selecionados portadores de PCA e outras cardiopatias congênitas e estruturais. Sua aplicação pode

\section{ABSTRACT}

Percutaneous Closure of Congenital and Structural Heart Defects with the Amplatzer Duct Occluder II $^{\mathrm{TM}}$

Background: A new generation of Amplatzer devices with lower profile and greater flexibility has been recently developed for percutaneous occlusion of the patent ductus arteriosus (PDA). In this paper, the use of this new device, the Amplatzer Duct Occludder II ${ }^{\mathrm{TM}}$ (ADO II) for percutaneous closure of the PDA as well as for the treatment of other congenital and structural heart defects is reported, assessing its safety and efficacy. Methods: Longitudinal and observational study of a cohort of patients with congenital and structural heart defects treated with ADO II between October 2009 and August 2011. Patients weighing less than $5 \mathrm{~kg}$ and PDAs > $5.5 \mathrm{~mm}$ and/or type B were excluded. The device was implanted using the antegrade or retrograde approach. The waist was $2 \mathrm{~mm}$ larger than the defect and the length of the prosthesis was $4 \mathrm{~mm}$ for shorter lesions (less than 6-8 mm). Results: Twenty-eight patients with a median age and weight of 2 years and $11.7 \mathrm{~kg}$, respectively, were included. Twenty-three had a PDA with a mean minimal diameter of $2.3 \pm 0.8 \mathrm{~mm}$. The remainder had muscular ventricular septal defect (1), pulmonary arteriovenous fistula (1), ruptured aneurysm of the sinus of Valsalva (1), and univentricular heart in the early postoperative period of bidirectional cavopulmonary anastomosis with residual antegrade pulmonary flow (2). The device was successfully implanted in all patients, except for an infant weighing $8 \mathrm{~kg}$ with a long, tortuous and oblique PDA with a diameter of $2.8 \mathrm{~mm}$ and a shallow ampulla. There was total occlusion of the defects in all patients. One patient with univentricular heart and right isomerism died due to complications not related to the procedure. Conclusions: ADO II proved to be

\footnotetext{
1 Hospital do Coração da Associação do Sanatório Sírio - São Paulo, SP, Brasil.

2 Instituto Dante Pazzanese de Cardiologia - São Paulo, SP, Brasil. 3 Hospital Unimed (CIAS) - Vitória, ES, Brasil.

Correspondência: Carlos A. C. Pedra. Instituto Dante Pazzanese de Cardiologia - Av. Dr. Dante Pazzanese, 500 - 14음 andar Ibirapuera - São Paulo, SP, Brasil - CEP 04012-180

E-mail: cacpedra@uol.com.br

Recebido em: 4/9/2011 • Aceito em: 16/11/2011
} 
ter limitações em algumas configurações anatômicas de PCA em lactentes menores.

DESCRITORES: Cardiopatias congênitas. Canal arterial. Cateterismo cardíaco. Próteses e implantes.

D iversas próteses foram desenvolvidas para o fechamento percutâneo da persistência do canal arterial (PCA) desde o primeiro relato feito por Portsmann et al. ${ }^{1}$, em 1967, utilizando plug vascular de ivalon. Hoje em dia, os dispositivos mais utilizados para oclusão de PCA são as molas de liberação controlada ou não-controlada e a prótese Amplatzer Duct Occluder $^{\text {TM }}$ (AGA Medical, Golden Valley, Estados Unidos)..$^{2-7}$ No entanto, em decorrência da variabilidade anatômica da PCA (como se fossem as "impressões digitais", próprias de cada paciente), nenhuma dessas próteses se mostrou versátil o suficiente para ser aplicada universalmente para todos os tipos e tamanhos. Por isso, inúmeras técnicas e dispositivos são empregados, com resultados, de modo geral, muito bons. ${ }^{2-7}$ Entretanto, possíveis complicações, como embolização e protrusão para a artéria pulmonar esquerda ou para a aorta, podem resultar de escolhas inadequadas de dispositivos para a anatomia subjacente. Algumas anatomias são mais desafiadoras, como os canais dos tipos C e B, especialmente em pequenos lactentes. Além disso, do ponto de vista técnico, algumas dificuldades são observadas de acordo com a estratégia e o dispositivo utilizado, incluindo a liberação não-controlada de molas implantadas por via retrógada, e o uso de bainhas longas de maior perfil e de sistemas mais rígidos de implante. Tais limitações, ainda que menores, podem resultar em maior manipulação intracardía$\mathrm{ca}$, aumento do tempo de procedimento e fluoroscopia com maior radiação. ${ }^{2}$ Pelos motivos expostos, a introdução de novas próteses flexíveis com sistemas de implante controlado, de baixo perfil, utilizadas por via anterógrada ou retrógrada pode ajudar a ampliar o espectro de indicação do procedimento para lactentes mais jovens, a aumentar as taxas de sucesso do implante e de oclusão, e a minimizar as dificuldades técnicas e complicações do procedimento.

Uma nova geração de próteses para a oclusão de PCA, Amplatzer Duct Occluder II ${ }^{\mathrm{TM}}$ (ADO II), foi desenvolvida, com base nas limitações descritas. A experiência inicial com esse dispositivo, tanto mundial como brasileira, é animadora. ${ }^{8-14}$ Além disso, a prótese ADO II também tem sido utilizada para oclusão de outras lesões e defeitos intra e extracardíacos, incluindo anastomoses de Blalock-Taussig ${ }^{15}$, comunicações interventriculares perimembranosas ${ }^{16}$, musculares ${ }^{16,17}$ ou residuais pós-cirúrgicas ${ }^{18}$, fistulas coronárias ${ }^{19}$ e leaks paravalvares. ${ }^{20}$ a flexible, safe and effective device for the percutaneous treatment of infants, children and selected adults with PDA and other congenital and structural heart defects. Its use may have limitations for some anatomical PDA configurations in smaller infants.

KEY-WORDS: Heart defects, congenital. Ductus arteriosus. Heart catheterization. Prostheses and implants.

Neste estudo, descrevemos nossa experiência com o uso dessa nova prótese para o fechamento percutâneo de PCA e de outros defeitos congênitos e estruturais, discutindo suas vantagens, limitações, segurança e eficácia.

\section{MÉTODOS}

\section{Desenho do estudo}

Estudo descritivo longitudinal observacional de uma coorte de pacientes submetidos a fechamento percutâneo do PCA e de outros defeitos congênitos e estruturais com a prótese ADO II. A coleta de dados foi realizada de forma retrospectiva, por meio da análise de prontuários. Foram coletados dados demográficos, clínicos, cirúrgicos, ecocardiográficos, hemodinâmicos, e relativos à técnica de fechamento.

\section{Pacientes}

Entre outubro de 2009 e agosto de 2011, 28 pacientes com mediana de idade de 2 anos $(0,3$ a 40 anos) e de peso de $11,7 \mathrm{~kg}(5,3 \mathrm{~kg}$ a $70 \mathrm{~kg})$ foram submetidos a oclusão percutânea de defeitos congênitos ou estruturais com a prótese ADO II em três centros de referência brasileiros. Desse total, 23 apresentavam PCA com repercussão hemodinâmica com aumento do ventrículo esquerdo à ecocardiografia. Peso inferior a $5 \mathrm{~kg}$ e canais $>5,5 \mathrm{~mm}$, além de PCA do tipo B, foram considerados critérios de exclusão para o tratamento percutâneo de PCA com ADO II. Dos 23 pacientes com PCA, 10 tinham menos de 12 meses de idade e 10 pesavam menos de $10 \mathrm{~kg}$. Os 5 pacientes restantes tratados com ADO II foram: uma criança de 5 anos e $20 \mathrm{~kg}$ com comunicação interventricular muscular trabecular de $4 \mathrm{~mm}$ de diâmetro e sobrecarga ventricular esquerda à ecocardiografia; um adulto de 40 anos e $70 \mathrm{~kg}$ com aneurisma roto do seio de Valsalva direito para o ventrículo direito, de etiologia indefinida, evoluindo com quadro de insuficiência cardíaca congestiva e dilatação do ventrículo esquerdo; uma criança de 3 anos e 13 kg portadora de fístula arteriovenosa pulmonar diagnosticada dentro do laboratório de cateterismo quando ia ser submetida a oclusão percutânea da comunicação interatrial tipo ostium secundum por apresentar dessaturação sistêmica de oxigênio de causa silenciosa; e dois lactentes portadores de corações univentriculares previamente 
submetidos a bandagem da artéria pulmonar, evoluindo com síndrome de veia cava superior no pós-operatório precoce (entre 4 dias e 7 dias) de cirurgia de Glenn. Um deles tinha 6 meses e 5,3 kg e diagnóstico de atresia tricúspide e discordância ventriculoarterial. $\mathrm{O}$ outro, também de 6 meses, pesava $6 \mathrm{~kg}$ e era portador de isomerismo atrial direito, defeito do septo atrioventricular total desbalanceado para a direita, dupla via de saída do ventrículo direito e estenose na anastomose de Glenn e na confluência das artérias pulmonares. Nos dois casos, o tronco da artéria pulmonar não foi totalmente ocluído cirurgicamente, permanecendo o aspecto da bandagem original. A falência na drenagem da veia cava superior foi atribuída à competição de fluxo pela via anterógrada pulmonar e possíveis estenoses no sistema de Glenn. Em todos os casos foi realizada uma oclusão teste da bandagem por cerca de 10 minutos com cateteres-balão tipo Berman ${ }^{\mathrm{TM}}$ (Critikon, Inc., Tampa, Estados Unidos) para averiguar a tolerância à oclusão da via de saída.

\section{Dispositivo e sistema de entrega}

A prótese ADO II é constituída de uma malha ultrafina, densa e com múltiplas camadas de nitinol, que forma dois discos simétricos de retenção, ambos de baixo perfil e articulados, conectados a uma cintura central (Figura 1). As conexões entre os discos e a cintura central são flexíveis, permitindo articulação entre esses componentes. ${ }^{8-14} \mathrm{~A}$ prótese é bem flexível e se alonga para se acomodar à anatomia subjacente. Não há retalhos de poliéster em seu interior. O poder oclusor da prótese se dá pelas 6 barreiras de nitinol ( 2 no disco distal, 2 na cintura e 2 no disco proximal). O disco proximal conecta-se ao cabo liberador por sistema de parafuso e rosca, comum a toda linha Amplatzer $^{\mathrm{TM}}$. O cabo liberador é manufaturado em aço

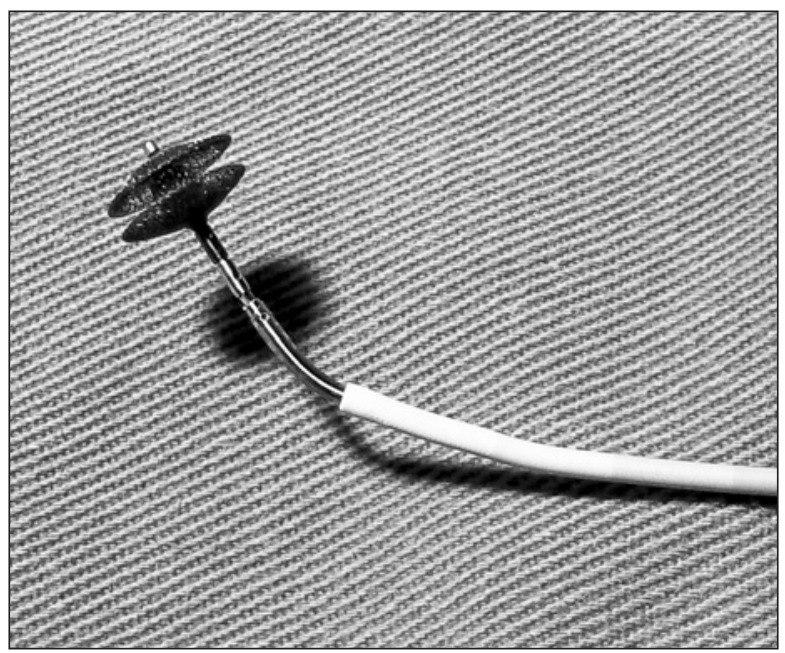

Figura 1 - Prótese Amplatzer Duct Occluder II ${ }^{\mathrm{TM}}$ (ADO II) $4 \times 4 \mathrm{~mm}$ montada em seu sistema de entrega e carregada no cateter terapêutico Judkins $^{\mathrm{TM}}$ de coronária direita $5 \mathrm{~F}$, demonstrando seu baixo perfil. inoxidável, sendo mais fino e flexível que os outros cabos feitos para conexão de outras próteses Amplatzer $^{\mathrm{TM}}$. A prótese ADO II está disponível no mercado nos tamanhos $3 \mathrm{~mm}, 4 \mathrm{~mm}, 5 \mathrm{~mm}$ e $6 \mathrm{~mm}$, correspondentes ao diâmetro de sua cintura central. O comprimento é de $4 \mathrm{~mm}$ ou $6 \mathrm{~mm}$. Os discos são $6 \mathrm{~mm}$ maiores que a cintura central. Os sistemas ou cateteres de entrega TorqVue LPTM (AGA Medical, Golden Valley, Estados Unidos) $4 \mathrm{~F}$ e $5 \mathrm{~F}$ são flexíveis e de menor perfil ( $\mathrm{LP}=$ low profile).

A escolha da prótese ADO II para oclusão de PCA baseou-se em seu tipo anatômico ${ }^{21}$, no menor diâmetro, no diâmetro da ampola e no comprimento. Uma tabela de recomendações para seleção do tamanho do dispositivo é fornecida pelo fabricante, mas de modo geral o tamanho da cintura da prótese escolhida foi de $1 \mathrm{~mm}$ a $2 \mathrm{~mm}$ maior que o diâmetro mínimo do canal arterial e seu comprimento foi de $4 \mathrm{~mm}$ naqueles com PCA de comprimento inferior a $6-8 \mathrm{~mm}$ e de $6 \mathrm{~mm}$ nos de comprimento maior. Procurou-se deixar um disco e a cintura central do lado aórtico, por ser o sistema de maior pressão. Procurou-se assegurar que o disco aórtico coubesse dentro da ampola. Como já comentado, canais $>5,5 \mathrm{~mm}$ e do tipo B foram considerados critérios de exclusão para o tratamento percutâneo de PCA com ADO II.

Nos outros defeitos, foram utilizados critérios de escolha de tamanhos do dispositivo semelhantes: o diâmetro da prótese ADO II foi cerca de 1-2 mm maior que o defeito e o comprimento foi de $4 \mathrm{~mm}$ para as lesões curtas e de $6 \mathrm{~mm}$ para as lesões mais longas.

\section{Procedimentos}

Em todos os casos, foi assinado o termo de consentimento livre e esclarecido pelos pacientes, pais ou responsáveis. Os procedimentos foram realizados sob anestesia geral. O acesso vascular (veia femoral ou jugular interna e artéria femoral) variou de acordo com a doença de base e a estratégia e a técnica a serem utilizadas. Heparina (100 U/kg; máximo de $5.000 \mathrm{UI})$ foi administrada seguida de antibioticoterapia profilática com cefalosporinas de primeira geração. Cateterismos cardíacos direito e esquerdo rotineiros foram praticados antes das angiografias e da oclusão dos defeitos. As angiografias foram obtidas em projeções ajustadas para ótima visibilização dos defeitos em questão e estruturas adjacentes.

Para os casos de oclusão de PCA foram obtidos acessos arterial e venoso femoral. Angiografia na aorta descendente em perfil esquerdo foi realizada com cateter pig-tail posicionado no istmo aórtico. Os canais arteriais foram classificados segundo os critérios angiográficos de Krichenko et al. ${ }^{21}$ e medidos em vários pontos: diâmetro mínimo, diâmetro da ampola aórtica e comprimento. Para canais $>2,5 \mathrm{~mm}$ e/ou mais longos e tortuosos, a técnica utilizada para o implante foi a anterógrada, semelhante àquela descrita previamente 
para a prótese ADO de primeira geração ${ }^{2,3}$ (Figura 2). Para os canais menores, com ampolas aórticas mais profundas e amplas, o implante foi feito por via retrógrada (Figura 3), utilizando-se a sombra traqueal como referência para a ancoragem do disco pulmonar. Uma pequena injeção manual de contraste pelo braço lateral da bainha TorqVue ${ }^{T M}$ foi realizada antes da liberação da prótese nos casos de implante retrógrado. As manobras clássicas para o implante de próteses Amplatzer $^{\mathrm{TM}}$, como empurrar o cabo liberador e puxar a bainha, foram empregadas de forma rotineira nos procedimentos. Entretanto, no caso do implante de ADO II, pela maior maleabilidade e menor robustez, essas manobras foram realizadas com maior delicadeza a fim de evitar a passagem total e inadvertida da prótese de um lado para o outro do canal arterial. O mesmo cuidado no implante foi tomado para os outros procedimentos descritos a seguir.

A oclusão da comunicação interventricular muscular foi realizada sob monitoração de ecocardiografia transesofágica (ETE). A comunicação interventricular foi cruzada retrogradamente e uma alça arteriovenosa foi estabelecida com um guia laçado no átrio direito. O dispositivo foi implantado por via venosa anterógrada, utilizando-se a veia jugular interna (Figura 4), de acordo com as técnicas já descritas. ${ }^{22}$ Optou-se pelo acesso anterógrado pela presença de trabécula septomarginal muito espessada ao redor do defeito, o que poderia atrapalhar o correto posicionamento e a estabilização da prótese se a via retrógada tivesse sido utilizada. Neste caso, uma bainha Cordis Bright Tip ${ }^{\mathrm{TM}}$ (Johnson
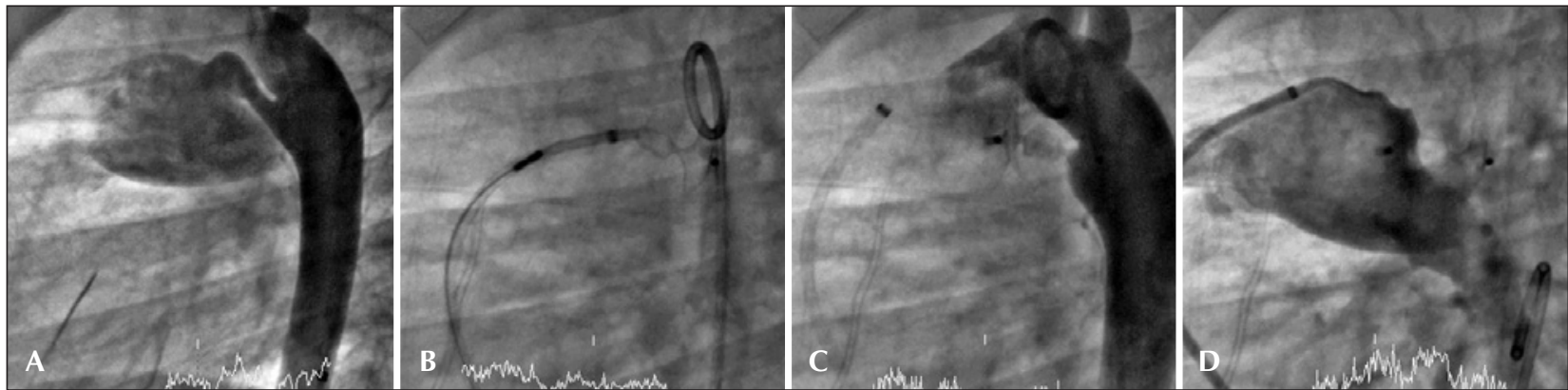

Figura 2 - Angiografias em perfil esquerdo, com discreta angulação cranial. Em A, persistência do canal arterial tortuoso do tipo E, sem constrições no trajeto que mede cerca de $3 \mathrm{~mm}$. Em B, posicionamento do disco aórtico e cintura central da prótese Amplatzer Duct Occluder II $^{\mathrm{TM}}$ (ADO II) $5 \times 6 \mathrm{~mm}$, por via anterógrada. Em C, angiografia aórtica após liberação da prótese, evidenciando posicionamento adequado, sem protrusão e obstruções na aorta. Em D, angiografia pulmonar demonstrando artéria pulmonar esquerda livre de estenoses.
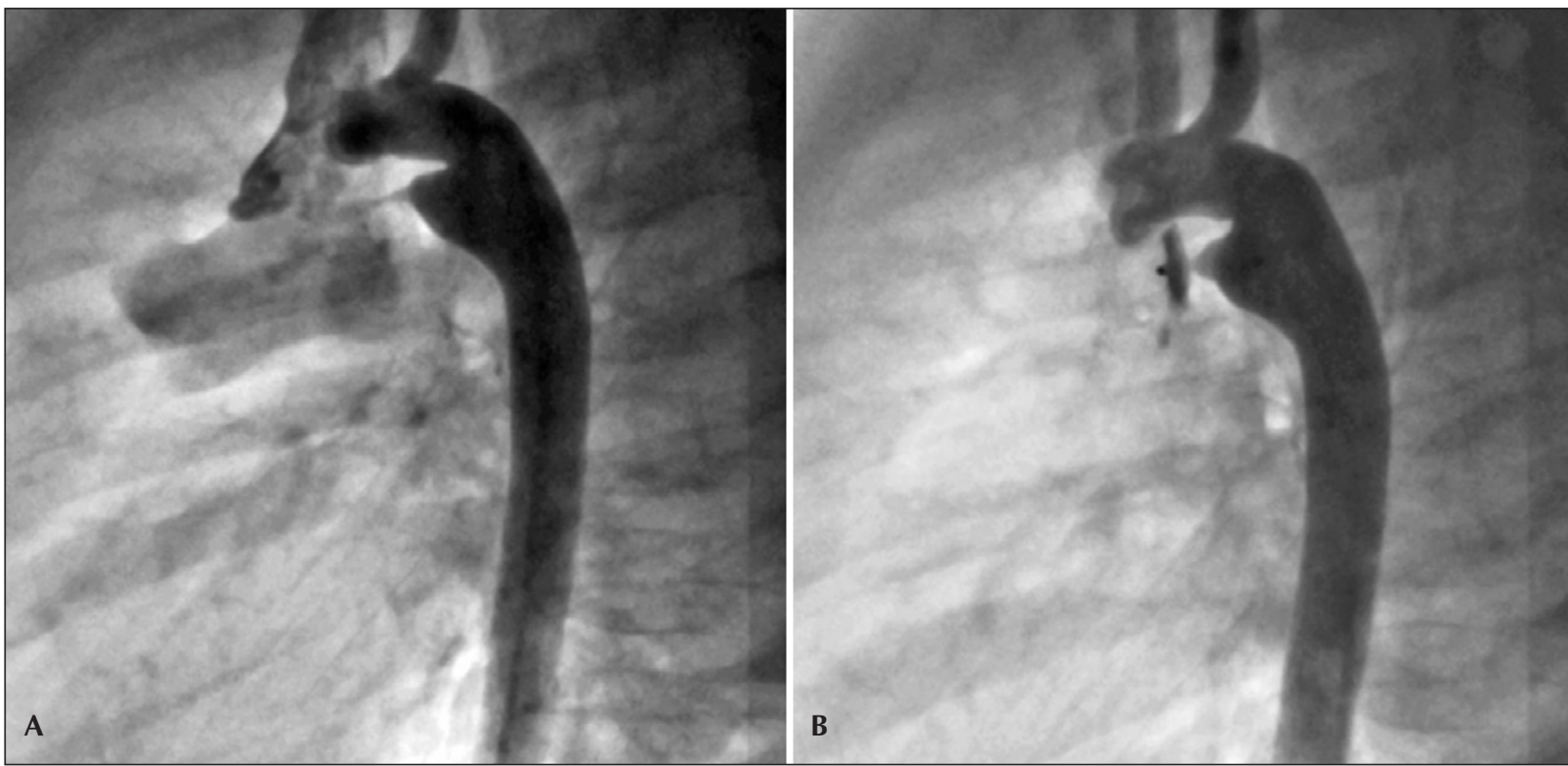

Figura 3 - Aortografias em perfil esquerdo. Em A, persistência do canal arterial do tipo A de 1,8 mm de diâmetro mínimo, com ampola aórtica profunda, e istmo e aorta descendente de calibres normais. Em B, posicionamento adequado da prótese Amplatzer Duct Occluder II ${ }^{\mathrm{TM}}$ (ADO II) $4 \times 4 \mathrm{~mm}$ após implante por via retrógrada, sem fluxo residual ou coarctação da aorta. 
\& Johnson Interventional Systems Co., New Jersey, Estados Unidos) $5 \mathrm{~F}$ de $45 \mathrm{~cm}$ de comprimento foi utilizada como sistema de entrega. No paciente com aneurisma roto do seio de Valsalva (seio direito para o ventrículo direito), o procedimento foi realizado tam- bém sob a monitoração de ETE. O orifício foi cruzado por via retrógrada (arterial) com o auxílio de um fio-guia hidrofílico, sendo o disco distal da prótese e a cintura central abertos no interior do ventrículo direito e o disco proximal deixado no assoalho do seio (Figura 5),

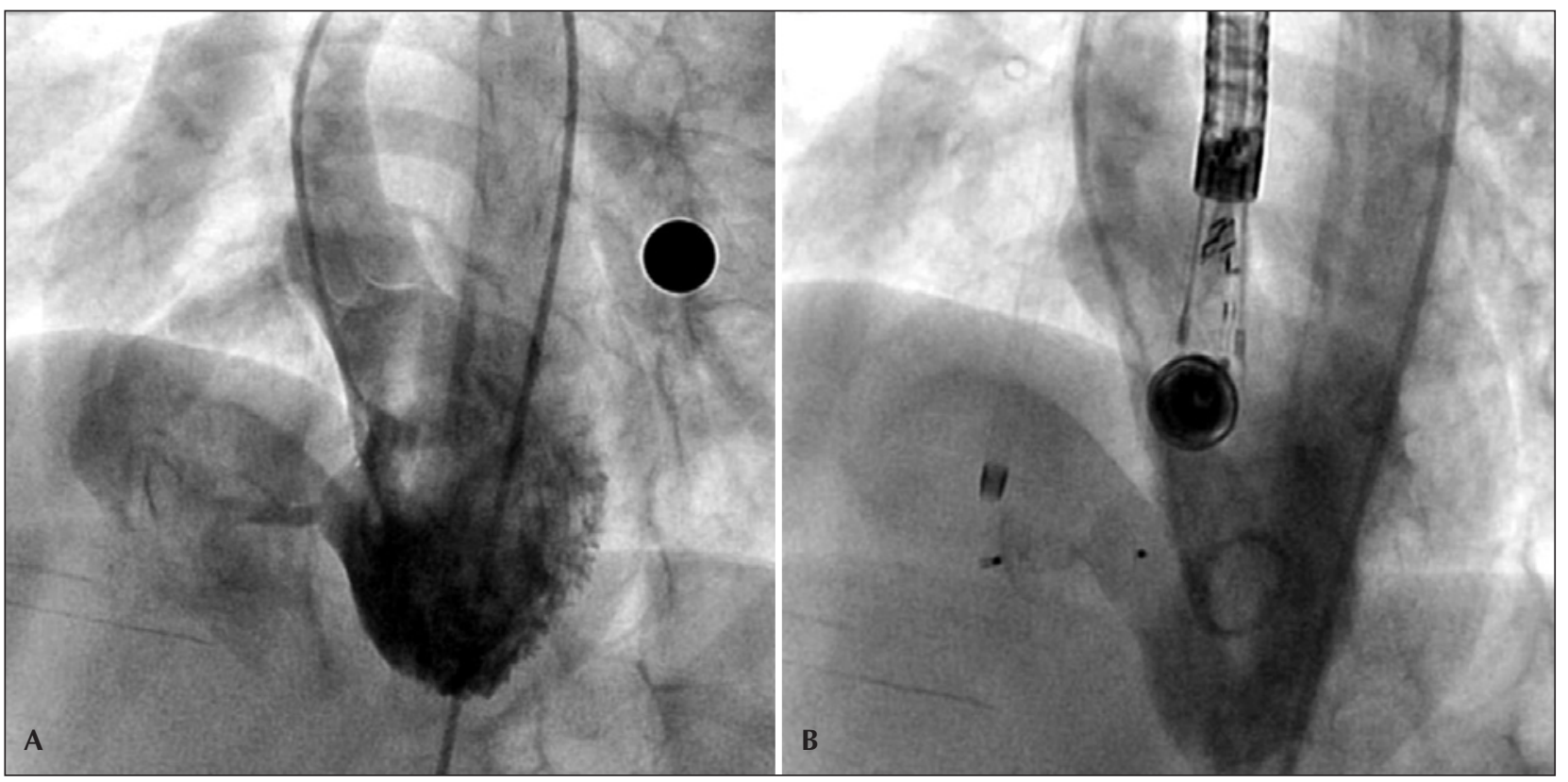

Figura 4 - Ventriculografias esquerdas em projeção hepatoclavicular. Em A, comunicação interventricular muscular média trabecular de cerca de $4 \mathrm{~mm}$ de diâmetro, com fluxo direcionado da esquerda para a direita e dilatação ventricular esquerda. Em B, prótese Amplatzer Duct Occluder II ${ }^{\mathrm{TM}}$ (ADO II) $6 \times 6 \mathrm{~mm}$ com posicionamento adequado após implante por via anterógrada, com mínimo fluxo residual pelo interior da malha imediatamente após a liberação.
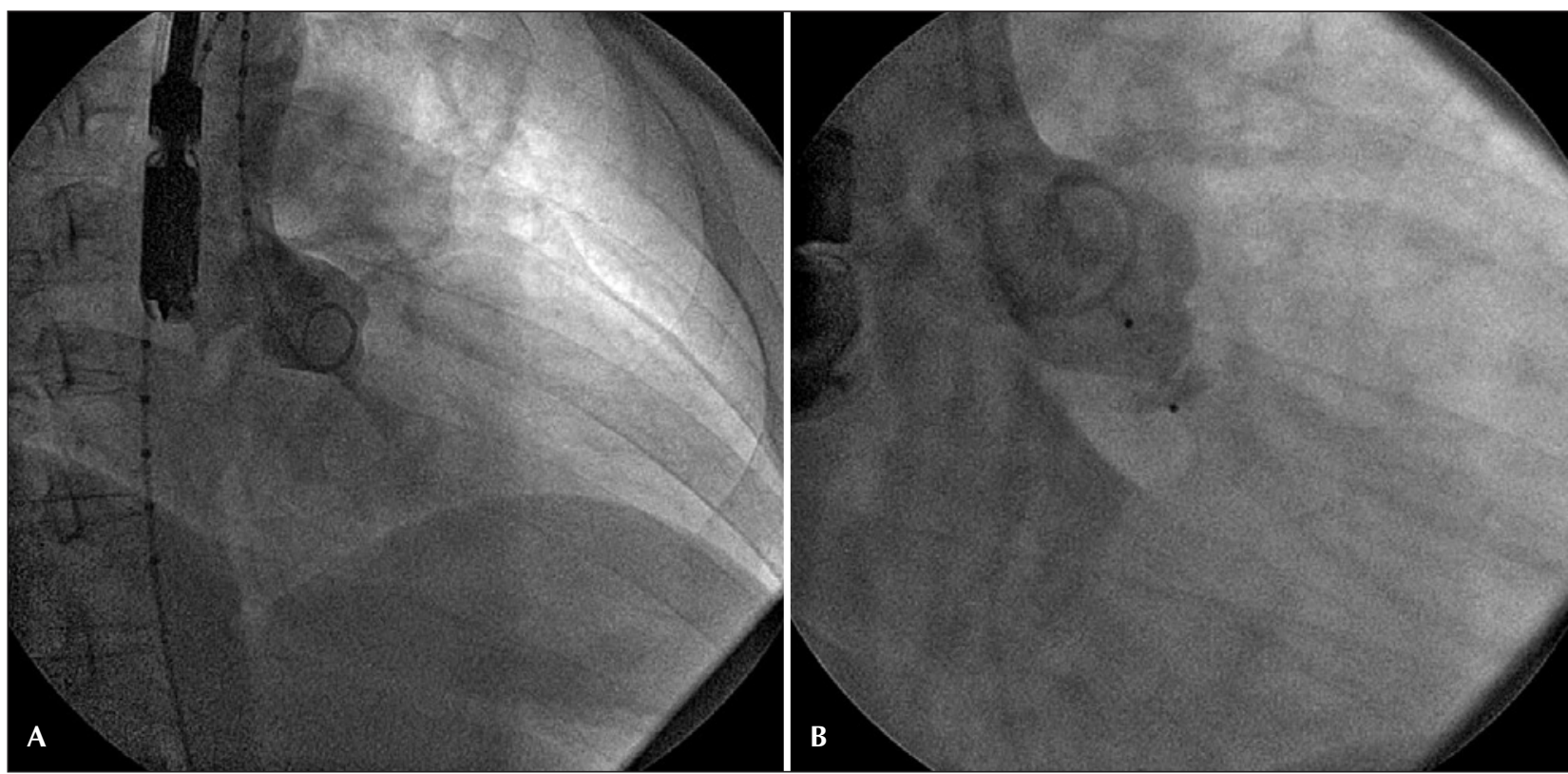

Figura 5 - Aortografias em oblíqua anterior direita. Em A, aneurisma roto do seio de Valsalva direito comunicando a raiz da aorta ao ventrículo direito, com comunicação medindo $2,8 \mathrm{~mm}$. Em B, prótese Amplatzer Duct Occluder II ${ }^{\mathrm{TM}}$ (ADO II) 5 x $4 \mathrm{~mm}$ bem posicionada após implante por via retrógrada, sem fluxo residual. 
não sendo necessário o estabelecimento de alça arteriovenosa, comumente utilizada nesse procedimento. ${ }^{23,24} \mathrm{Um}$ cateter-guia terapêutico $6 \mathrm{~F}$ multipurpose foi utilizado como sistema de entrega. Outra via de acesso arterial $6 \mathrm{~F}$ foi utilizada para angiografias de controle com cateter pig-tail. No paciente portador de fístula arteriovenosa pulmonar com vaso nutridor proveniente da artéria pulmonar direita (Figura 6), a prótese foi avançada por via femoral dentro de um sistema TorqVue LPTM $5 \mathrm{~F}$ passado coaxialmente por dentro de uma bainha longa $7 \mathrm{~F}$ posicionada na artéria pulmonar direita, de acordo com técnicas semelhantes já descritas. ${ }^{25,26}$ Tal artifício foi empregado para se obter maior estabilidade dos sistemas de entrega no local desejado durante o implante, além de possibilitar injeções de contraste pelo braço lateral da bainha longa $7 \mathrm{~F}$ para averiguação do posicionamento correto da prótese. A comunicação interatrial tipo ostium secundum de $11 \mathrm{~mm}$ foi ocluída posteriormente com a técnica habitual, empregando-se dispositivo Amplatzer Septal Occluder ${ }^{\mathrm{TM}}$ (ASO) de $14 \mathrm{~mm}$.

Nos 2 pacientes portadores de corações univentriculares no pós-operatório de cirurgia de Glenn, o fechamento da via de saída pulmonar no local da bandagem foi realizado pela via jugular interna direita com técnica semelhante às técnicas descritas anteriormente. ${ }^{27} \mathrm{~A}$ bandagem foi cruzada da artéria pulmonar para o ventrículo principal com cateter Judkins de coronária direita e fio-guia hidrofílico. Sobre um fio-guia de troca, o cateter TorqVue LPTM $5 \mathrm{~F}$ foi avançado da jugular interna até a cavidade ventricular. $\mathrm{O}$ disco distal e a cintura central foram deixados abaixo da bandagem. Os folhetos da valva pulmonar foram capturados pelo disco distal a fim de que permanecessem abertos, evitando-se um espaço morto entre a valva e a prótese, o que poderia facilitar a formação de trombos no local. Apenas o disco proximal ficou posicionado do lado da artéria pulmonar dentro do sistema de Glenn. As angiografias de controle para correto posicionamento da prótese foram realizadas por meio de cateter angiográfico avançado por via venosa femoral até o ventrículo principal (Figura 7). No paciente portador de estenose na anastomose de Glenn e na confluência entre as artérias pulmonares, estas foram posteriormente tratadas com angioplastia com balão (Cristal Balloon ${ }^{\text {TM }} 10$ x $20 \mathrm{~mm}$, Balt Extrusion, Montmorency, França) na anastomose seguida de implante de stent Palmaz Genesis $^{\text {TM }} 1910$ (Johnson \& Johnson Interventional Systems Co., New Jersey, Estados Unidos) montado sobre o mesmo Cristal Balloon ${ }^{\text {TM }} 10 \times 20 \mathrm{~mm}$ através da bainha Bright Tip $^{\text {TM }}$ (Johnson \& Johnson Interventional Systems Co., New Jersey, Estados Unidos) $8 \mathrm{~F}$ avançada pela veia jugular interna e cava superior até o local estenótico (Figura 8).

Em todos os casos, angiografias de controle foram realizadas após o posicionamento e a liberação da prótese para avaliação de possíveis fluxos residuais e obstruções vasculares adjacentes causadas pelos discos da prótese. Recuo pressórico com cateter de furo terminal também foi realizado no interior do vaso tratado, a fim de averiguar possíveis obstruções vasculares decorrentes do implante do dispositivo. No dia seguinte ao procedimento, todos os pacientes foram avaliados clinicamente e por meio de ecocardiografia.

O acompanhamento ambulatorial foi feito de forma rotineira, com avaliações clínicas e ecocardiográficas periódicas, de acordo com cada enfermidade de base.

\section{Análise estatística}

Os resultados estão apresentados em frequência, média, mediana e desvio padrão, conforme a distribuição da amostra.
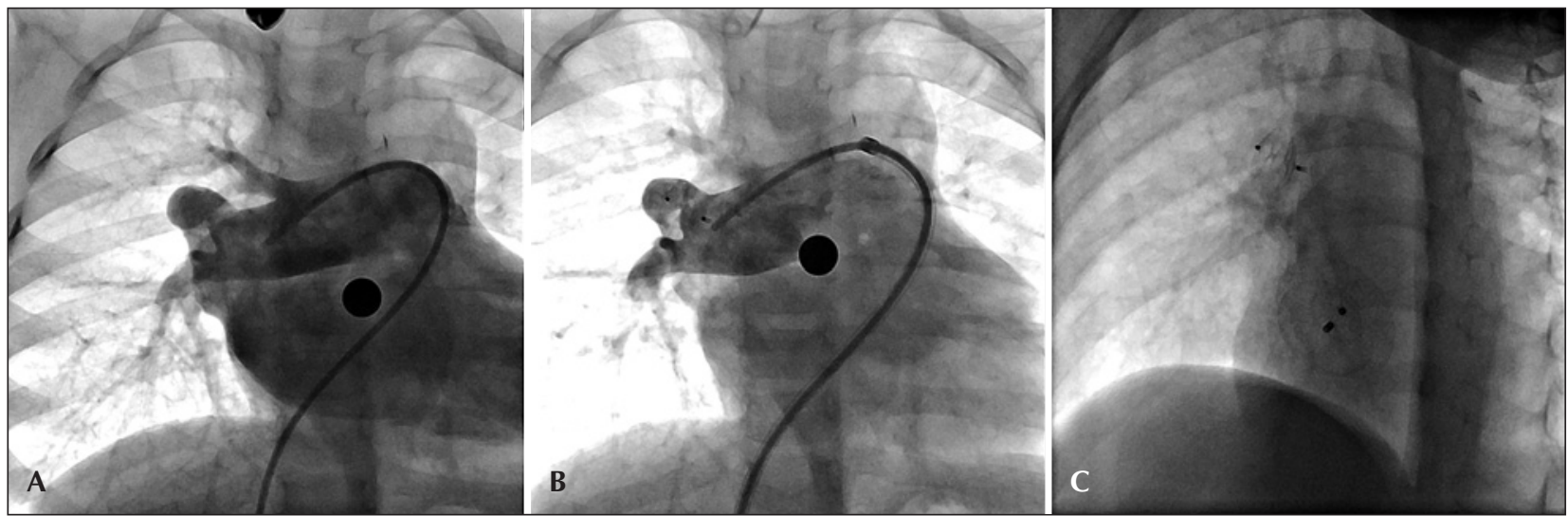

Figura 6 - Angiografias pulmonares em projeção póstero-anterior: Em A, artéria pulmonar direita dilatada. Do ramo para o lobo médio origina-se fístula arteriovenosa de cerca de $4 \mathrm{~mm}$ de diâmetro, com rápida opacificação da veia pulmonar superior direita e do átrio esquerdo. Em B, angiografia de controle após a liberação do dispositivo, evidenciando posicionamento adequado da prótese Amplatzer Duct Occluder II ${ }^{\mathrm{TM}}$ (ADO II) $6 \times 4 \mathrm{~mm}$, com disco proximal aposto à parede da artéria e o restante no interior da fístula, com mínimo fluxo residual imediato. Em C, radioscopia do tórax em projeção hepatoclavicular, mostrando ADO II e Amplatzer Septal Occluder ${ }^{\mathrm{TM}}$ (ASO) após oclusão da comunicação interatrial. 


\section{RESULTADOS}

\section{Pacientes com persistência do canal aterial}

Segundo a classificação angiográfica de Krichenko, 17 pacientes eram portadores de PCA do tipo A, 1 do tipo C, 1 do tipo D, e 4 do tipo E. A média do diâmetro mínimo da PCA determinado pela angiografia foi de $2,3 \pm 0,8 \mathrm{~mm}$ e a média do tamanho da cintura da prótese ADO II escolhida foi de 4,1 $\pm 0,9 \mathrm{~mm}$. Nenhum paciente apresentava hipertensão pulmonar significativa. A prótese foi implantada com sucesso em 22 dos 23 pacientes, sendo por via retrógrada em 9 pacientes. O paciente no qual não se obteve êxito no implante tinha 1 ano de idade e $8,5 \mathrm{~kg}$ e era portador de PCA longo e tortuoso do tipo E, com 2,8 $\mathrm{mm}$ no menor diâmetro e $12 \mathrm{~mm}$ de extensão. O canal possuía

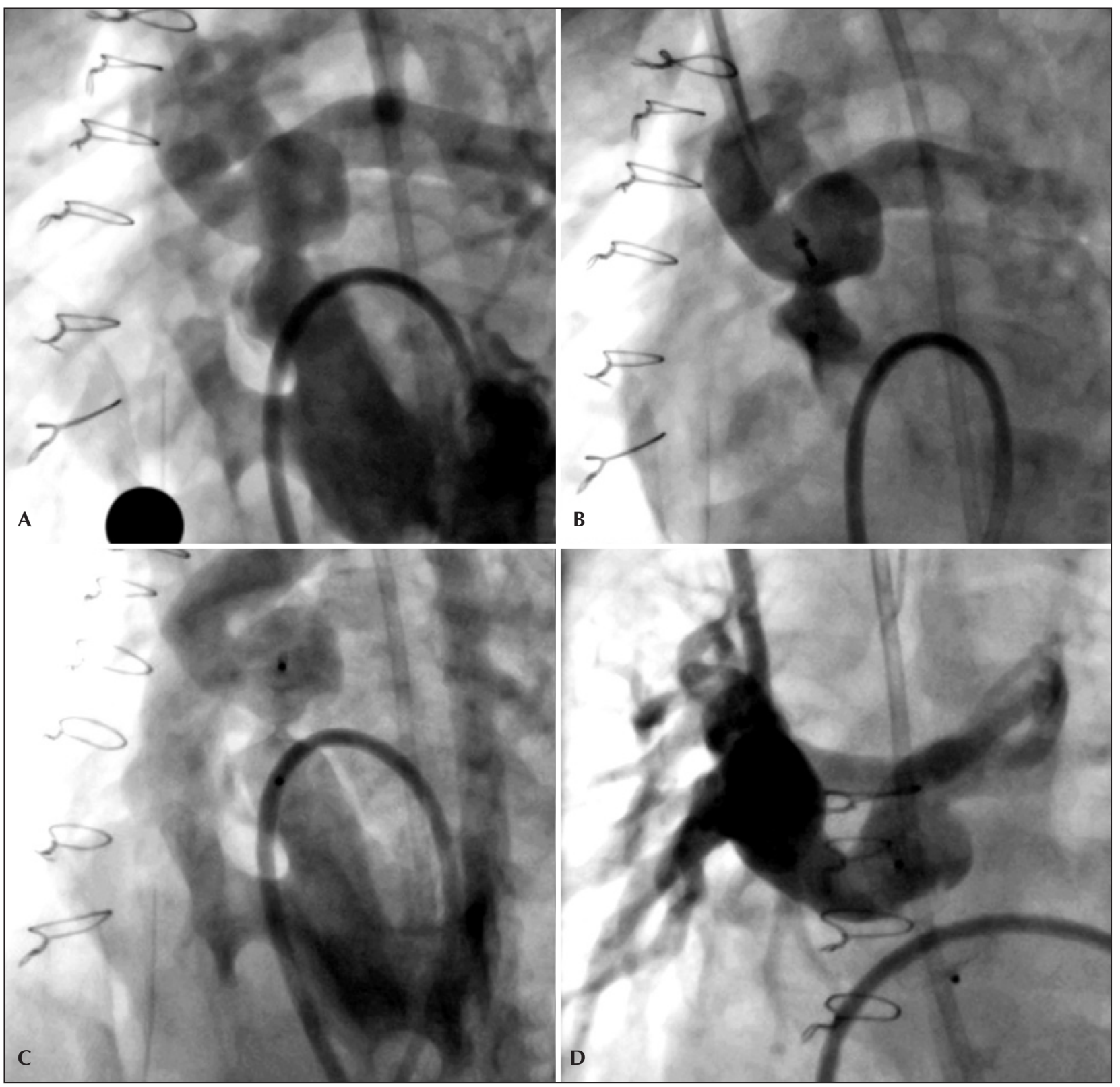

Figura 7 - Angiografias em projeção oblíqua anterior esquerda cranial. Em A, ventriculografia esquerda evidenciando atresia tricúspide, discordância ventriculoarterial e comunicação interventricular não-restritiva, com fluxo anterógrado pulmonar significativo através da bandagem do tronco da artéria pulmonar. Em B, angiografia pulmonar pelo braço lateral de uma bainha curta 7 F, mostrando prótese Amplatzer Duct Occluder II ${ }^{\mathrm{TM}}$ (ADO II) implantada anterogradamente pela veia cava superior, ainda conectada ao cabo liberador, com disco distal bem posicionado na porção inferior da bandagem e disco proximal no assoalho das artérias pulmonares. Em C, ventriculografia esquerda mostrando mínimo fluxo residual através da malha da prótese imediatamente após a liberação da ADO II 6 × $4 \mathrm{~mm}$. Em D, angiografia pulmonar após liberação final do dispositivo confirma ótimo posicionamento e ausência de estenoses dos ramos pulmonares. 

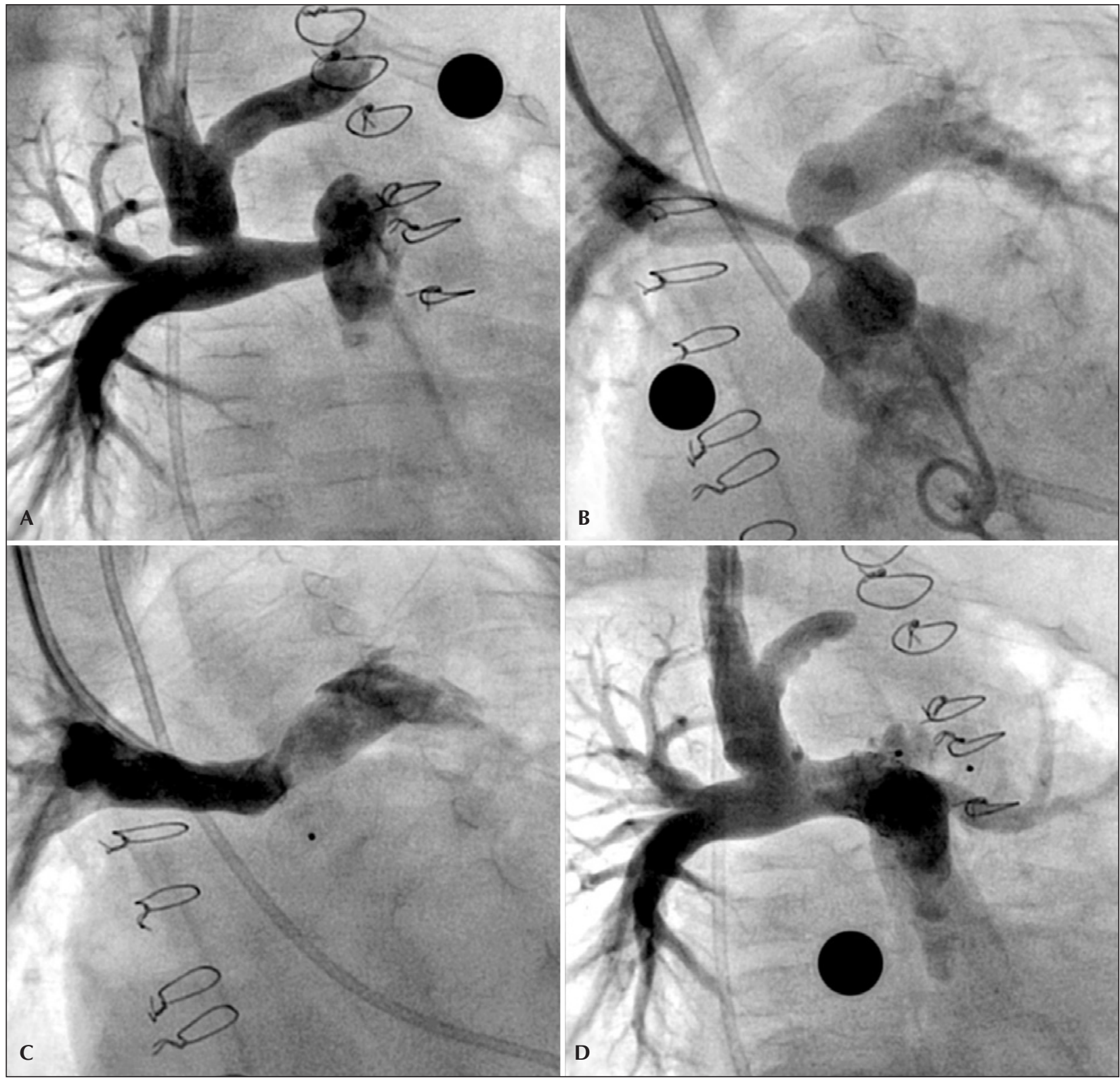

Figura 8 - Em A, angiografia na veia cava superior em oblíqua anterior direita caudal, com estenose discreta da anastomose de Glenn e na confluência das artérias pulmonares. Em B, ventriculografia direita em oblíqua anterior esquerda cranial, mostrando fluxo anterógrado significativo pela bandagem do tronco pulmonar e migração distal da bandagem, resultando em estenose significativa na confluência das artérias pulmonares. Em C, angiografia pulmonar em projeção oblíqua anterior esquerda cranial após liberação da prótese Amplatzer Duct Occluder II ${ }^{\mathrm{TM}}$ (ADO II) $6 \times 4 \mathrm{~mm}$. A prótese está bem ancorada na bandagem, com discreta protrusão do disco proximal Em D, angiografia na veia cava superior em oblíqua anterior direita caudal após término dos procedimentos. Observa-se nítida melhora no local da anastomose da veia cava superior com a artéria pulmonar direita, posicionamento adequado da prótese e ampliação significativa no local da confluência das artérias pulmonares após implante de stent; entretanto, o fluxo para o pulmão esquerdo encontra-se lentificado.

ampola aórtica rasa de cerca de $6 \mathrm{~mm}$, trajeto oblíquo, e formava um ângulo agudo de aproximadamente 60 graus com o istmo aórtico, acarretando protrusão significativa do disco esquerdo para a aorta, a despeito de inúmeras tentativas de reposicionamento (Figura 9). Nesse caso, optou-se pela troca do dispositivo por um Nit-Occlud ${ }^{\text {TM }}$ PDA (PFM Medical, Colônia, Alemanha) $7 \times 6 \mathrm{~mm}$, que ficou bem posicionado e ocluiu a PCA com sucesso. Não foram observadas complicações relacionadas à oclusão da PCA em nenhum paciente. Ecocardiografia transtorácica (ETT) realizada no dia seguinte ao procedimento revelou oclusão completa dos defeitos nos 23 pacientes e fluxos de velocidades normais na aorta e na artéria pulmonar esquerda. Todos os pacientes tiveram alta hospitalar após 24 horas de internação, sem apresentar intercorrências clínicas. Em 

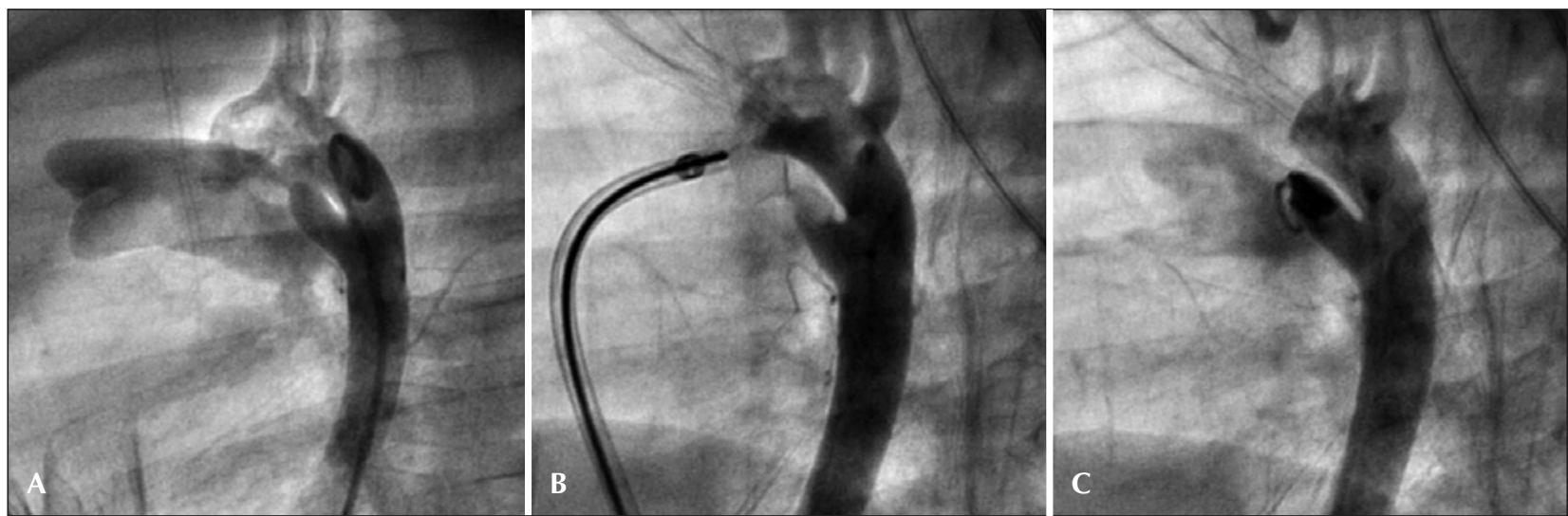

Figura 9 - Aortografias em perfil esquerdo. Em A, persistência do canal arterial do tipo E com diâmetro mínimo de 2,8 mm, com intenso fluxo esquerda-direita. Em B, protrusão significativa do disco distal da prótese Amplatzer Duct Occluder II ${ }^{\mathrm{TM}}$ (ADO II) durante implante por via anterógrada, causando coarctação iatrogênica da aorta. Em C, prótese Nit Occlud ${ }^{\mathrm{TM}} 7 \times 6 \mathrm{~mm}$ bem posicionada dentro da ampola, com discreto fluxo residual imediato.

seguimento médio de $9 \pm 3$ meses, todos os pacientes permanecem sem fluxo residual à ecocardiografia.

\section{Pacientes com outros defeitos}

A comunicação interventricular muscular de $4 \mathrm{~mm}$ de diâmetro foi ocluída com êxito por via anterógrada com uma prótese ADO II 5 × 6 mm. As ecocardiografias intraprocedimento e no dia seguinte confirmaram correto posicionamento da prótese e ausência de fluxo residual. O paciente teve alta hospitalar no dia seguinte, com ritmo sinusal ao traçado do eletrocardiograma. Após 9 meses de seguimento, a ecocardiografia revelou normalização das dimensões do ventrículo esquerdo e ausência de fluxo residual pela prótese.

No paciente portador de aneurisma roto do seio de Valsalva, a angiografia na raiz da aorta revelou um orifício medindo cerca de $3 \mathrm{~mm}$ de diâmetro. A prótese ADO II escolhida foi a 4 × $4 \mathrm{~mm}$, sendo implantada sem dificuldades. A angiografia aórtica e a ETE após o implante confirmaram ótimo posicionamento do dispositivo e ausência de fluxo residual. Não houve comprometimento do movimento dos folhetos da valva aórtica. Alta hospitalar foi dada após 24 horas. Após um ano de acompanhamento, o paciente encontra-se assintomático e sem medicação e a ecocardiografia mostra oclusão do defeito, funcionamento normal da valva aórtica e ventrículo esquerdo de dimensões normais.

A fístula arteriovenosa pulmonar era tortuosa e media, em seu menor diâmetro, cerca de $4 \mathrm{~mm}$. O defeito foi ocluído com prótese ADO II 6 × 4 mm, posicionada com sucesso pelo sistema TorqVue LPTM 5 F. Não houve formação completa dos discos, que assumiram configuração globosa, exercendo força radial na parede do vaso. A opção pelo uso de ADO II neste caso decorreu da indisponibilidade de uso de outros dispositivos no momento da intervenção. A angiografia de controle realizada 10 minutos após a liberação evidenciou pequena passagem de contraste através da malha da prótese. Houve normalização imediata dos níveis de saturação sistêmica de oxigênio (de 85\% para 98\%). A comunicação interatrial tipo ostium secundum associada de $11 \mathrm{~mm}$ foi ocluída com dispositivo ASO $14 \mathrm{~mm}$ sem intercorrências, com auxílio de ETE, que não evidenciou fluxo residual ao final do procedimento. $\mathrm{O}$ paciente recebeu alta no dia seguinte. Seis meses após, os níveis de saturação permanecem normais e o paciente encontra-se assintomático, sem fluxo residual pela comunicação interatrial à ecocardiografia.

Os 2 pacientes com coração univentricular tiveram a via de saída pulmonar ocluída com êxito com dispositivos ADO II $6 \times 4 \mathrm{~mm}$, entregues através de sistema TorqVue LPTM 5 F. Após o implante do dispositivo, observou-se queda da saturação sistêmica de oxigênio de $80-85 \%$ para $65-75 \%$ e da pressão média pulmonar, de 18-22 $\mathrm{mmHg}$ para 15-17 $\mathrm{mmHg}$, com estabilidade hemodinâmica. Não foram evidenciados gradientes de pressão dos ramos pulmonares causados por protrusão do disco proximal (pulmonar) da prótese após recuo pressórico. Na ventriculografia de controle, houve passagem de pequena quantidade de contraste pelo interior da prótese, que se encontrava bem posicionada em todos os casos. Naquele caso no qual havia estenose prévia no local de confluência das artérias, o stent implantado se sobrepôs ao disco proximal (pulmonar) da prótese ADO II implantada anteriormente. O paciente com atresia tricúspide com vasos transpostos evoluiu com melhora gradual do quadro de síndrome de cava superior, sendo extubado 4 dias após o procedimento. O paciente com isomerismo direito evoluiu com piora progressiva dos níveis de saturação, coagulopatia e redução da perfusão no pulmão esquerdo, culminando com óbito 48 horas após. Não foi permitida a realização de autópsia. Retrospectivamente, as angiografias durante o procedi- 
mento levantaram a hipótese de estenose intraparenquimatosa de veias pulmonares esquerdas.

\section{DISCUSSÃO}

Este trabalho é o segundo no Brasil a relatar o uso de ADO II para o tratamento percutâneo de $\mathrm{PCA}^{8}$ e o primeiro a relatar seu uso para oclusão de outros defeitos congênitos e estruturais. De modo geral, o dispositivo mostrou-se de fácil manipulação, versátil, seguro e com altas taxas de oclusão. As modificações notadas na prótese ADO II em relação à geração anterior (designada ADO I) compreendem, principalmente, sua conformação em dois discos flexíveis e articulados em dois pontos e seu menor perfil, decorrente da malha ultrafina de nitinol e ausência de tecido no interior do dispositivo. O sistema de entrega também foi modificado, proporcionando menor perfil e maior flexibilidade. Dessa forma, tornou-se possível a abordagem de canais arteriais antes mais desafiadores, como aqueles de trajeto mais longo, principalmente em lactentes ${ }^{8-14}$, e o emprego da via retrógada para oclusão. Tais vantagens ficaram bem evidenciadas neste estudo, já que 10 dos pacientes com PCA tinham menos de 12 meses de idade e em 9 procedimentos o dispositivo foi implantado retrogradamente, reduzindo a manipulação intracardíaca e o tempo de procedimento. Entretanto, em um paciente desta casuística o posicionamento do dispositivo foi considerado subótimo em decorrência de protrusão discreta do disco esquerdo para a aorta, sendo necessária sua remoção. Neste caso, o canal, além de longo (> $15 \mathrm{~mm}$ ) e tortuoso, possuía orientação oblíqua e ampola rasa e de diâmetro $<6 \mathrm{~mm}$, não acomodando o disco distal. Acreditamos que esstes casos devam ser abordados com próteses com disco distal retilíneo, de menor diâmetro e com maior poder de compactação. Provavelmente a nova versão da prótese ADO II (ADO II AS), que possui essas características $^{28}$, poderia ter sido útil naquele caso, que foi resolvido satisfatoriamente com o implante de outro tipo de prótese (Nit-Occlud ${ }^{\mathrm{TM}}$ ). Isso denota a necessidade de o intervencionista manter-se aberto a múltiplas possibilidades e de ter sempre essa gama de opções disponível em seu estoque de materiais.

O perfil mais baixo da prótese ADO II permitiu a entrega em sistemas de menor calibre TorqVue LPTM $4 \mathrm{~F}$ ou $5 \mathrm{~F}$, desenvolvidos pelo mesmo fabricante. Esse fato, somado a sua conformação em duplo-disco, possibilitou seu uso por via retrógrada para a oclusão dos canais arteriais de anatomia mais favorável, sem que houvesse incremento do risco de complicação do acesso vascular. A via retrógrada para o implante tornou o procedimento mais simples e rápido e excluiu a necessidade do trajeto intracardíaco de fios-guia, cateteres e sistemas de liberação, como visto em 9 pacientes em nossa casuística. Entretanto, reconhecemos que a maioria dos pacientes deste estudo submetidos ao implante retrógrado de ADO II possuía canais de pe- queno calibre, que poderiam ter sido abordados com o emprego de molas de Gianturco, como descrito em experiências prévias $^{6}$, com menores custos. A facilidade e a versatilidade no uso de ADO II através da via arterial foram demonstradas também na descrição de um caso de implante do dispositivo por meio de acesso arterial braquial esquerdo com introdutor 5 F. ${ }^{29}$ Tratava-se de um adulto de 66 anos com quadro de insuficiência cardíaca congestiva decorrente de canal arterial grande e verticalizado, em que o ângulo de ataque para o tratamento se mostrou mais adequado quando proveniente da artéria subclávia esquerda, sem que complicações do acesso vascular fossem constatadas. Acreditamos que a via anterógrada deva ser usada para o tratamento dos canais arteriais maiores ou de abordagem mais complexa, nos quais o perfeito posicionamento do disco aórtico é mais desafiador. Incluem-se nesses casos os canais arteriais do tipo A mais angulados ou dos tipos $\mathrm{C}$ e $\mathrm{E}$, especialmente em lactentes de menor peso. Nessas situações, parece ser mais sábio reservar a via arterial para a realização de aortografias de controle durante o fechamento do canal arterial e a liberação da prótese pelo lado pulmonar. Entre todos os tipos anatômicos de canal arterial segundo a classificação de Krichenko, talvez o mais apropriado para uso de ADO II seja o do tipo D ou o do tipo A com ampola ampla e profunda.

Apesar da limitação do fabricante ao uso da prótese em pacientes com PCA com peso superior a $5 \mathrm{~kg}$, já existe experiência comprovada de seu uso em pacientes menores. Um estudo francês relatou o implante da prótese ADO II em 58 recém-nascidos e lactentes com peso inferior a $6 \mathrm{~kg}$, tendo o menor paciente 3,4 kg. ${ }^{30}$ Mesmo apresentando taxas de complicação pouco maiores que a casuística cirúrgica deste estudo, essa prótese pode ser considerada uma alternativa nas situações em que o estado clínico do paciente seja uma contraindicação à cirurgia. Sem dúvida, maiores estudos são necessários para se definir claramente quais critérios utilizar na seleção de ADO II para fechamento do canal arterial. Neste momento acreditamos que as melhores indicações sejam as seguintes ${ }^{31}$ : canais cônicos (tipo A de Kirchenko ${ }^{21}$ ), cujo diâmetro da aorta descendente, logo abaixo da inserção do canal, seja superior a $8 \mathrm{~mm}$, pelo risco de protrusão do disco aórtico; canais tubulares (tipo $\mathrm{C}$ de Kirchenko ${ }^{21}$ ) com mais de $6 \mathrm{~mm}$ de comprimento; e canais de anatomia complexa, com múltiplas tortuosidades ou constrições (tipo $E$ de Krichenko $\left.{ }^{21}\right)$. Nos pacientes com peso inferior a $15 \mathrm{~kg}$, parece mais prudente utilizar a via anterógrada para o implante, sendo puncionados os acessos arterial e venoso, mesmo que haja a necessidade da utilização de um cateter-laço para cruzar o canal através de seu lado pulmonar. ${ }^{31}$ Como possíveis complicações do uso da prótese ADO II, além de protrusão para a aorta ou artéria pulmonar esquerda, especialmente em lactentes com menos de $6 \mathrm{~kg}$ com grandes canais $(>4 \mathrm{~mm})^{30}$, a literatura também cita deslocamento tardio do dispo- 
sitivo e acotovelamento do disco esquerdo ${ }^{32,33}$, não observados nesta casuística.

Por causa de suas características vantajosas (flexibilidade, baixo perfil, segurança no implante e altas taxas de oclusão), a prótese ADO II passou a ser utilizada em outras cardiopatias congênitas e estruturais, como mostram a literatura ${ }^{15-20}$ e este estudo. A opção pelo uso não-convencional de ADO II nesta casuística facilitou a abordagem dos defeitos, uma vez que um sistema de entrega de menor calibre pôde ser usado com maior facilidade para alcançar o posicionamento ideal de todo o conjunto, além de acarretar menor risco de lesão vascular. Empregando-se técnicas semelhantes às utilizadas para outros dispositivos, conseguimos ocluir com certa facilidade, segurança e eficácia lesões congênitas e estruturais variadas. A comunicação interventricular muscular foi fechada com sucesso através do acesso anterógrado do sistema de entrega $5 \mathrm{~F}$, que foi avançado com facilidade através da espessa trabécula septo-marginalis ao redor do defeito. O sistema de entrega de baixo perfil, como o da prótese ADO II, facilita o fechamento de comunicações interventriculares musculares de até $5 \mathrm{~mm}$, inclusive em pequenos lactentes ${ }^{16-18}$, já que pode ser utilizado até mesmo por via retrógrada. Nesse sentido, o uso da via retrógrada para o fechamento do aneurisma roto de seio de Valsalva em um paciente desta casuística facilitou muito o procedimento, que habitualmente é realizado com próteses de maior perfil por via anterógrada após estabelecimento de uma alça arteriovenosa. ${ }^{23,24}$ No caso do tratamento da fístula arteriovenosa pulmonar, apesar de essa lesão ser mais comumente abordada com um Plug Vascular Amplatzer ${ }^{\mathrm{TM}},{ }^{25}$ de menor custo, tal dispositivo não estava disponível para uso no momento do procedimento. Além disso, a anatomia nos pareceu mais apropriada para o uso da prótese ADO II. O fechamento com molas não nos pareceu prudente, pelo calibre aumentado da fístula e seu alto fluxo, que acarreta risco elevado de embolização do dispositivo. Apesar de a prótese ADO II ter assumido formato globoso e comprimento maior que o habitual em decorrência dessa conformação espacial, cuidados foram tomados para evitar estenose iatrogênica do ramo arterial ou venoso pulmonar adjacente. Finalmente, nos casos de oclusão da via de saída pulmonar nos pontos de bandagem dos corações univentriculares, a prótese ADO II mostrou-se eficaz e de fácil manipulação. Apresenta vantagens em relação a outras próteses ${ }^{27}$ por ter menor perfil e por apresentar pequenas conexões finas articuladas intercalando a cintura central e os dois discos, em que a mais proximal cavalga a bandagem, deixando o disco que se conecta com o cabo bem aposto no assoalho da artéria pulmonar. Isso ajuda a evitar protrusões locais, o que poderia implicar estenose ou oclusão da artéria pulmonar adjacente. O óbito registrado em um dos pacientes decorreu das condições clínicas desfavoráveis prévias à intervenção e à doença de base (isomerismo direito com possível estenose de veias pulmonares), não estando relacionado ao fechamento da via de saída anterógrada pulmonar por si só. Esse paciente poderia ter apresentado melhor prognóstico se a cirurgia de Glenn não tivesse sido realizada ou se a via de saída não tivesse sido ocluída.

\section{CONCLUSÕES}

A prótese ADO II mostrou-se versátil, segura e eficaz para oclusão de PCA de até $5 \mathrm{~mm}$ de diâmetro (excluindo-se a do tipo B), tanto por via anterógrada como por via retrógada, inclusive em pequenos lactentes. Também mostrou-se útil para a abordagem de outros defeitos congênitos e estruturais. Seu menor perfil e a maior flexibilidade do sistema são vantagens inequívocas. Entretanto seus discos de diâmetro significativamente maior que a cintura central podem ser problemáticos em pequenos lactentes com canais oblíquos e de ampola rasa de pequeno diâmetro. O uso contínuo da prótese ADO II em um número maior de pacientes com PCA e com outras cardiopatias congênitas ou estruturais é necessário para a determinação do real papel desse novo dispositivo na oclusão percutânea dessas lesões.

\section{CONFLITO DE INTERESSES}

Carlos A. C. Pedra é consultor da AGA Medical. Os demais autores declaram não haver conflito de interesses relacionado a este manuscrito.

\section{REFERÊNCIAS}

1. Porstmann W, Wierny $\mathrm{L}$, Warneke $\mathrm{H}$. Closure of the persistent ductus arteriosus without thoracotomy. Ger Med Mon. 1967; 12(6):259-61.

2. Pass RH, Hijazi Z, Hsu DT, Lewis V, Hellenbrand WE. Multicenter USA Amplatzer patent ductus arteriosus occlusion device trial: initial and one-year results. J Am Coll Cardiol. 2004;44(3):513-9.

3. Simões LC, Pedra CA, Esteves CA, Camargo R, Braga SL, Loureiro $\mathrm{P}$, et al. Percutaneous closure of ductus arteriosus with the amplatzer prosthesis. The Brazilian experience. Arq Bras Cardiol. 2001;77(6):520-31.

4. Haddad J, Lima Filho MO, Figueiredo GL, Nazzeta HE, Osterne ECV. Oclusão percutânea da persistência do canal arterial. Rev Bras Cardiol Invasiva. 2005;13(3):206-18.

5. Arnoni DG, Peña JJS, Fontes VF, Braga SLN, Esteves CA, Ferreira WP, et al. Oclusão percutânea do canal arterial $>3 \mathrm{~mm}$ com auxílio do biótomo. Rev Bras Cardiol Invasiva. 2007;15(2): 134-40.

6. Pedra CAC, Esteves CA, Braga SLN, Pedra SRFF, Pontes Júnior SC, Silva MAP, et al. Oclusão percutânea do pequeno canal arterial com molas de Gianturco: impacto da otimização da seleção das molas e dos pacientes e da não-tolerância ao fluxo residual significativo imediato nos resultados. Rev Bras Cardiol Invasiva. 2008;16(1):86-90.

7. Lopes RM, Gonçalves FM, Barbosa MR, Martins CN, Vrandecic MO. Oclusão percutânea do canal arterial com molas de liberação controlada. Rev Bras Cardiol Invasiva. 2010;18(1): 68-73. 
8. Queiroz FJAC, Simões LC, Queiroz DSC, Ramos S, Silva JFA, Mattos R. Tratamento percutâneo do canal arterial com a prótese Amplatzer Duct Occluder II (ADO II): nova opção para um antigo defeito. Rev Bras Cardiol Invasiva. 2010;18(2): 204-11.

9. Forsey J, Kenny D, Morgan G, Hayes A, Turner M, Tometzki $A$, et al. Clinical experience with the new Amplatzer Ductal Occluder II for closure of the persistent arterial duct. Catheter Cardiovasc Interv. 2009;74(4):615-23.

10. Thanopoulos B, Eleftherakis N, Tzannos K, Stefanadis C, Giannopoulos A. Further experience with catheter closure of patent ductus arteriosus using the new Amplatzer Duct Occluder in children. Am J Cardiol. 2010;105(7):1005-9.

11. Saliba Z, El-Rassi I, Abi-Warde MT, Chehab G, Daou L, Khater D, et al. The Amplatzer Duct Occluder II: a new device for percutaneous ductus arteriosus closure. J Interv Cardiol. 2009;22(6):496-502.

12. Dua J, Chessa M, Piazza L, Negura D, Micheletti A, Bussadori $C$, et al. Initial experience with the new Amplatzer Duct Occluder II. J Invasive Cardiol. 2009;21(8):401-5.

13. Bhole V, Miller P, Mehta C, Stumper O, Reinhardt Z, De Giovanni JV. Clinical evaluation of the new Amplatzer duct occluder II for patent arterial duct occlusion. Catheter Cardiovasc Interv. 2009;74(5):762-9.

14. Koh GT, Ai Mokthar S, Hamzah A, Kaur J. Transcatheter closure of patent ductus arteriosus and interruption of inferior vena cava with azygous continuation using an Amplatzer duct occluder II. Ann Pediatr Cardiol. 2009;2(2):159-61.

15. Kenny D, Walsh KP. Transcatheter occlusion of a classical BT shunt with the Amplatzer Duct Occluder II. Catheter Cardiovasc Interv. 2008;72(6):841-3.

16. Koneti NR, Penumatsa RR, Kanchi V, Arramraj SK, Bhupathiraju $S$. Retrograde transcatheter closure of ventricular septal defects in children using the Amplatzer Duct Occluder II. Catheter Cardiovasc Interv. 2011;77(2):252-9.

17. El Tahlawi M, Kammache I, Fraisse A. Ventricular septal defect closure in a small children with the Amplatzer Duct Occluder II. Catheter Cardiovasc Interv. 2011;77(2):268-71.

18. Ramakrishnan S, Saxena A, Choudhary SK. Residual VSD closure with an ADO II device in an infant. Congenit Heart Dis. $2011 ; 6(1): 60-3$.

19. Karagöz T, Yıldırım I, Celiker A. Transcatheter closure of coronary artery fistula with an Amplatzer Duct Occluder II in a symptomatic infant. Anadolu Kardiyol Derg. 2011;11(3): 274-5.

20. Kursaklioglu H, Barcin C, Iyisoy A, Baysan O, Celik T, Kose S. Percutaneous closure of mitral paravalvular leak via retrograde approach: with use of the Amplatzer Duct Occluder II and without a wire loop. Tex Heart Inst J. 2010;37(4):461-4.
21. Krichenko A, Benson LN, Burrows $P$, Möes CA, McLaughlin $\mathrm{P}$, Freedom RM. Angiographic classification of the isolated, persistently patent ductus arteriosus and implications for percutaneous catheter occlusion. Am J Cardiol. 1989;63(12): 877-80.

22. Pedra CAC, Pedra SRF, Pessotti C, Santana MVT, Jatene I, Shimoda $M$, et al. Fechamento percutâneo da comunicação interventricular muscular congênita. Rev Bras Cardiol Invasiva. 2008;16(2):218-24.

23. Szkutnik M, Kusa J, Glowacki J, Fiszer R, Bialkowski J. Transcatheter closure of ruptured sinus of valsalva aneurysms with an Amplatzer occluder. Rev Esp Cardiol. 2009;62(11): 1317-21.

24. Khoury A, Khatib I, Halabi M, Lorber A. Transcatheter closure of ruptured right-coronary aortic sinus fistula to right ventricle. Ann Pediatr Cardiol. 2010;3(2):178-80.

25. Peirone AR, Spillman A, Pedra C. Successful occlusion of multiple pulmonary arteriovenous fistulas using Amplatzer vascular plugs. J Invasive Cardiol. 2006;18(3):E121-3.

26. Pereira FL, Ribeiro MS, Costa RN, Braga SLN, Fontes VF, Pedra CAC. Experiência clínica com a utilização de plugs vasculares Amplatzer. Rev Bras Cardiol Invasiva. 2010;18(3): 327-36.

27. Pilla CB, Fontes VF, Pedra CA. Obliteration of a competitive forward flow from the ventricle after a bidirectional cavopulmonary shunt with an Amplatzer duct occluder. J Invasive Cardiol. 2003;15(2):98-101.

28. Bass JL, Wilson N. Transcatheter occlusion of the patent ductus arteriosus in infants - experimental testing of a new amplatzer device. Catheter Cardiovasc Interv. 2011 Jan 13. [Epub ahead of print]

29. Cassese S, Losi MA, Rapacciuolo A. Transradial approach for percutaneous closure of patent ductus arteriosus with the Amplatzer Duct Occluder II: a case report. Catheter Cardiovasc Interv. 2011;77(1):103-7.

30. Abadir S, Boudjemline Y, Rey C, Petit J, Sassolas F, Acar P, et al. Significant persistent ductus arteriosus in infants less or equal to $6 \mathrm{~kg}$ : percutaneous closure or surgery? Arch Cardiovasc Dis. 2009;102(6-7):533-40.

31. Venczelova Z, Tittel P, Masura J. The new Amplatzer Duct Occluder II: when is its use advantageous? Cardiol Young. 2011;21(5):495-504.

32. Barcin C, Kursaklioglu H. Editorial: late-term displacement of Amplatzer Duct Occluder II device following percutaneous closure of patent ductus arteriosus in an adult. J Interv Cardiol. 2010;23(4):368-9.

33. Beck C, Laser KT, Haas NA. Failure of the Amplatzer Ductal Occluder II: kinking of the aortic retention disk at 24 hours. Catheter Cardiovasc Interv. 2010;75(7):1100-3. 